\title{
Terre ! Une formation d'enseignants sur le dialogue créatif entre arts et sciences
}

\section{Soil! A teacher training course on the creative interplay between art and science}

\author{
Claire Calmet ${ }^{1}$, Elise Duc-Fortier ${ }^{2}$, Anaïs Tondeur $^{3}$ \\ ${ }^{1}$ professeur de SVT, collège Alain Fournier d'Orsay \\ ${ }^{2}$ directrice de l'association S[cube] \\ ${ }^{3}$ artiste
}

RÉSUMÉ. Cet article témoigne d'une formation destinée à des professeurs de collège de toutes disciplines, sur la thématique de l'usage des terres émergées. Cette formation, née de la rencontre entre une artiste et des médiateurs scientifiques, a proposé un parcours riche en expériences sensorielles, mêlant échanges avec artistes et chercheurs, pratique scientifique et création artistique.

ABSTRACT. This article describes a training course for secondary school teachers of all disciplines, on the subject of land use. This training, born from the encounter between an artist and scientific facilitators, proposed a journey rich in sensorial experiences, blending exchanges with artists and researchers, scientific practice and artistic creation.

MOTS-CLÉS. Formation, Enseignant, Interdisciplinaire, Sol, Usage des terres, Olfaction, Pétrichor, Sensorialité.

KEYWORDS. Training, Teacher, Interdisciplinary, Soil, Land use, Olfaction, Petrichor, Sensoriality.

De la rencontre entre l'artiste Anaïs Tondeur et l'équipe de l'association S[cube] - Partageons les sciences est né un parcours de formation à la croisée de l'art et des sciences, sur les enjeux de l'usage des sols. Tandis que S[cube] mettait sur pied en 2020, en écho au dernier rapport du GIEC, un dispositif d'enquête immersive sur l'usage des terres émergées, Anaïs Tondeur déployait depuis 2015 le protocole Pétrichor sur l'anthropisation des sols. Des préoccupations communes, des regards complémentaires et l'opportunité d'initier un public enseignant à une approche sensible des questions scientifiques ont donné vie à cette formation. La démarche de l'artiste a constitué le fil conducteur de la formation, émaillé par les éclairages scientifiques, les temps de création offerts aux participants et les moments d'échanges sur les pratiques pédagogiques mêlant arts et sciences.

\section{Aux sources de la formation : l'enquête Pétrichor de l'artiste Anaïs Tondeur}

Entremêlant fiction, recherches de terrain et alchimie, l'enquête Pétrichor se déploie à travers les sols urbains, de Paris aux Lilas, de Nogent-sur-Marne à Montreuil, de Montpellier à Aberdeen. Ce projet participatif mobilisant habitants, jardiniers et réfugiés, enseignants, chercheurs, étudiants et jeunes élèves se construit sur les traces du pétrichor - l'odeur si particulière du sol après la pluie, ouvrant un espace de réflexions et d'échanges sur nos façons de percevoir la ville. À l'issue de cette exploration de terrain, une installation révèle le processus de création interdisciplinaire et participatif.

Ce projet est d'abord une évocation du travail des chimistes du 19e siècle qui s'intéressaient par l'odorat aux boues de Paris, notamment les boues ferrugineuses situées sous et entre les pavés de la ville. Il est aussi un hommage aux scientifiques australiens qui découvrirent le pétrichor en 1964, et inventèrent ce néologisme issu de petra - la pierre - et ichor - le sang des dieux. Ces scientifiques 
furent mal compris. On crut que le pétrichor était une qualité intrinsèque des choses, et beaucoup de parfumeurs cherchèrent à imiter cette odeur « de terre après la pluie ». Mais le pétrichor est autre chose. Il désigne le système complexe par lequel la pluie et le sol interagissent pour produire une odeur. Il ne peut donc émerger que dans la relation entre le sol et le climat, et nous rappelle que cette interaction est présente partout sur terre, bien que l'on cherche à l'empêcher en ville du fait de l'importante densité de population, pour limiter le développement des «miasmes ».

Par une mise en fiction, en suggérant de sentir le sol, ce projet hybride invite à réfléchir au sol à partir de la notion de relation. Évoquer les « miasmes » et la « transpiration de la terre », c'est envisager les manières par lesquelles on se met en danger si l'on ne prend pas soin des sols. Ainsi, envisager les sols des villes en termes de relations auxquelles nous participons, c'est aussi réfléchir aux conditions de nos existences urbaines.

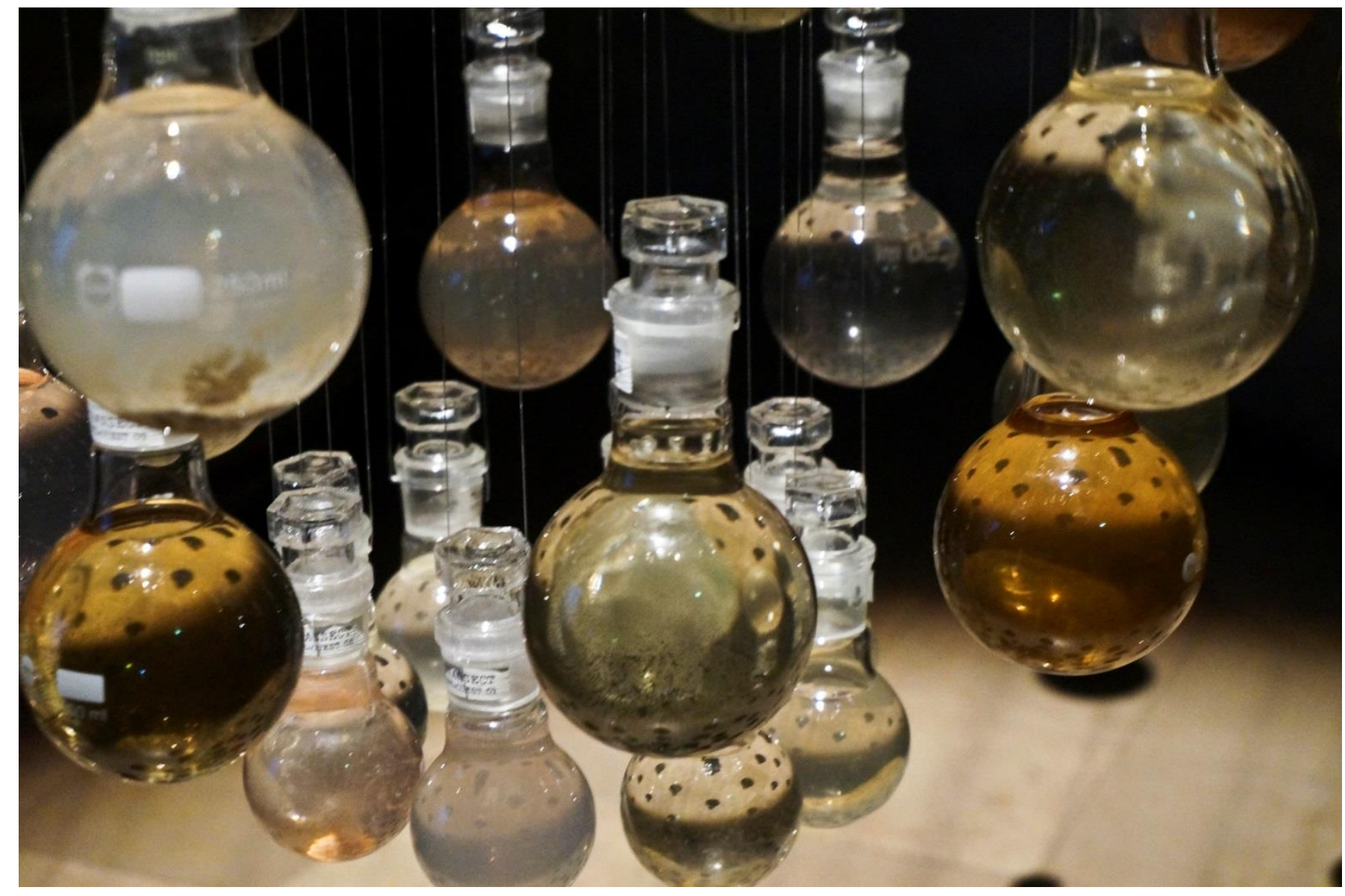

Vue de l'installation, Nuit Blanche, Paris 2016,

Pétrichor, Anaïs Tondeur \& Germain Meulemans

Cette installation a été présenté au Musée d'Art et d'histoire de St Denis, à la Nuit Blanche 2016 (Paris), au Centre Pompidou (Paris), au Centre Tignous d'Art contemporain, au Domaine Départemental de Chamarande, au laboratoire d'anthropologie Knowing from the Inside de Tim Ingold (Ecosse). 


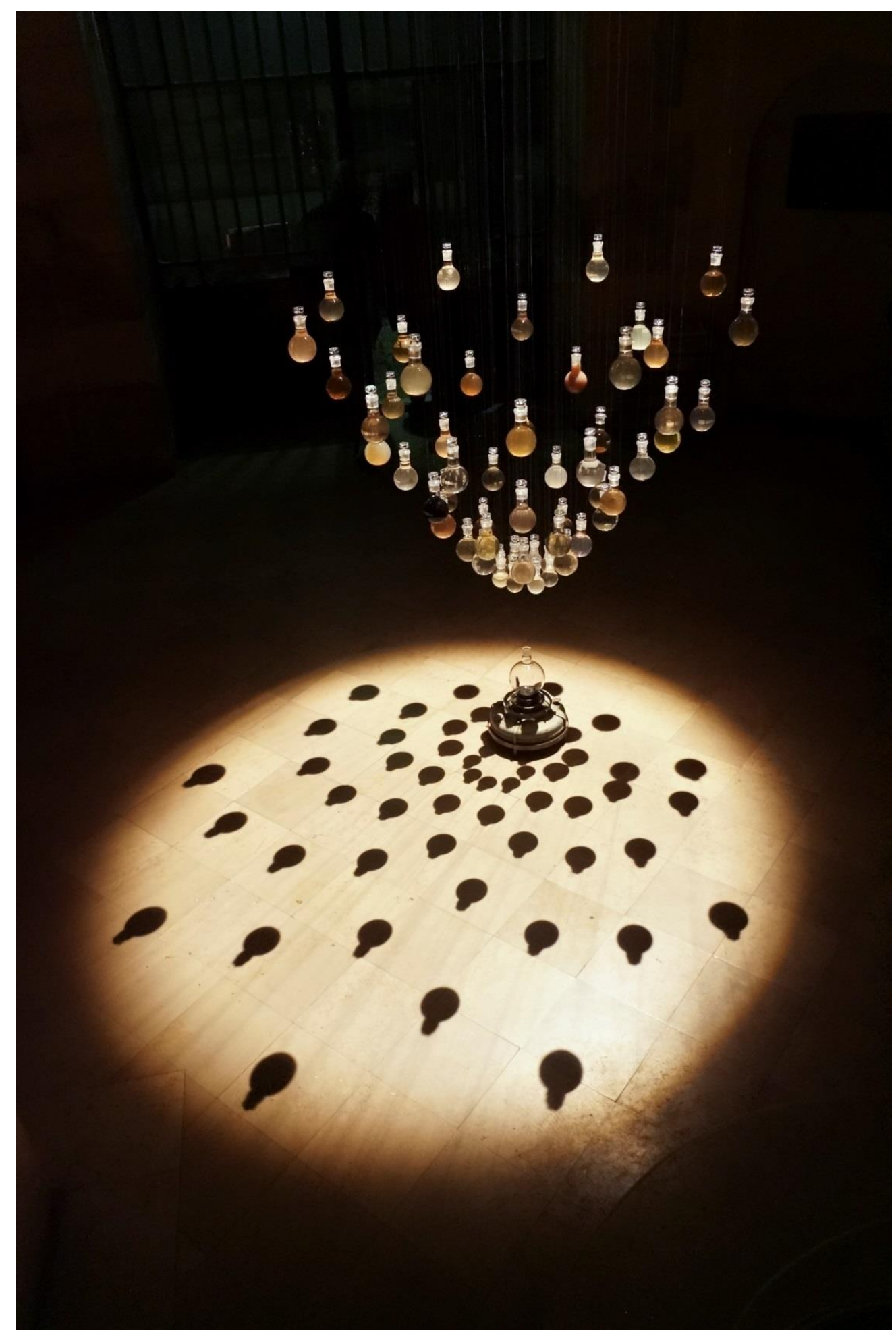

Vue de l'installation, Nuit Blanche, Paris 2016, Pétrichor, Anaïs Tondeur \& Germain Meulemans

Ce protocole a été conçu et développé par l'anthropologue Germain Meulemans et l'artiste Anaïs Tondeur dans le cadre d'une résidence transdisciplinaire au sein du Lab des cultures durables, initiée par COAL et le Domaine départemental de Chamarande autour de la thématique des sols, en 2015 et 2016 avec Yesenia Thibault-Picazo (designer multidisciplinaire), Marine Legrand (anthropologue) et Alan Vergnes (écologue) Marraine: Nathalie Blanc (Ladyss, CNRS) 
Comme le rappelle George Marshall ${ }^{1}$ dans son livre Le syndrome de l'autruche ${ }^{2}$, les discours strictement scientifiques sur les enjeux du développement durable ne sont pas suffisants pour amorcer et entretenir un engagement des individus et des groupes sociaux qui soit à la hauteur du défi. En effet, plus que nos connaissances et nos convictions, ce sont nos actes qui nous engagent, et ce d'autant plus que ces actes sont chargés émotionnellement. En conséquence, alors que l'éducation au développement durable a été mise en accord à la rentrée 2020 avec l'Agenda 2030 de l'ONU, les équipes pédagogiques doivent s'interroger sur les moyens les plus appropriés d'impliquer leurs élèves dans des actions engageantes sur ce thème.

Convaincus qu'une approche sensible participe de l'émotion qui habite les expériences que l'on garde au cœur (encadrés 3 et 4), l'équipe de l'association S[cube] et Anaïs Tondeur ont proposé aux professeurs en formation un parcours qui sollicitait leurs sens, comme en ont témoigné les participants (encadrés 1 et 5) : "Tous nos sens étaient convoqués, en particulier le sens olfactif, pour que l'étude des sols et de leurs usages prenne une dimension émotionnelle." Les éclairages scientifiques qui ont ponctué ce parcours ont nourri la pratique artistique des participants. Ils leur ont permis de découvrir des exemples de collaborations entre artistes et scientifiques telles les pièces de théâtre olfactif de Violaine de Carné (voir lien en fin d'article) et de s'approprier le dispositif d'enquêtes immersives $S[l a b]$ - Voyages en terres émergées (encadré 2) créé par l'association S[cube].

\section{À l'issue de la formation : des projets d'action culturelle}

Après deux journées riches d'expériences personnelles et collectives, d'échanges entre participants et avec les intervenants, les professeurs sont invités à concevoir des projets d'action culturelle inspirés par ce qu'ils ont vécu. Certains, venus en équipe pédagogique pluridisciplinaire avec un projet déjà bien construit, ont enrichi la réflexion du groupe. D'autres, venus en éclaireur, chercheront, au retour dans leur établissement, à partager leur expérience avec leurs collègues d'autres disciplines et à trouver avec eux un terrain de collaboration (encadrés 1 et 5). Afin de les accompagner dans la mise en œuvre en classe de projets faisant dialoguer les arts et les sciences, l'équipe qui a conçu et animé la formation reste à leur écoute et à leur disposition : mise en relation avec un.e artiste, des scientifiques et un lieu culturel, aide à la construction de leur projet d'action culturelle et à son déploiement en établissement et hors les murs. Pour que cette formation soit un commencement.

\section{Encadré 1}

\section{Témoignage de Djamila Gadouche, professeur en formation}

Professeur de SVT au collège Louis Paulhan de Sartrouville, je suis toujours en quête d'inspirations qui me rapprochent de mes collègues d'autres disciplines. Lorsque nous portons des regards croisés sur le monde, nous sommes en mesure de faire vivre à nos élèves des expériences pédagogiques qui transcendent les matières d'enseignement. Le stage "Arts et sciences, un dialogue créatif" auquel j'ai participé m'a permis d'envisager une thématique chère à ma discipline, celle de l'usage des sols, sous un angle absolument inattendu : tous nos sens étaient convoqués, en particulier le sens olfactif, pour que l'étude des sols et de leurs usages prenne une dimension émotionnelle. Lors de la première journée de

\footnotetext{
${ }^{1}$ Fondateur à Oxford du Climate Outreach and Information Network

${ }^{2}$ Le syndrome de l'autruche, pourquoi notre cerveau veut ignorer le changement climatique, Actes Sud, 2017
} 
stage, armée de petits récipients, d'une cuillère et de ma tablette pour prendre des photos, me voilà partie sous la neige recueillir des échantillons de sol. Collecter, observer, toucher, sentir, décrire. Plus tard, grâce au protocole proposé, je me retrouve dans ma cuisine. Après avoir emprunté une cafetière italienne, je « distille » mon échantillon de sol. Inspirée par l'odeur, je laisse mon imagination s'exprimer à travers mes mains en pétrissant un matériau pour donner forme à l'odeur, mais aussi à travers mes mots car l'odeur du sol me permet d'imaginer un récit.

De l'échantillonnage de sols sur le terrain à l'hydro-distillation des échantillons, de la formulation des sensations olfactives éveillées par l'odeur des hydrolats à l'évocation artistique du passé et du futur de l'environnement de collecte, le parcours proposé au cours du stage était inspiré par le travail de l'artiste Anaïs Tondeur (encadré 4), qui a su partager avec nous sa sensibilité. Ce parcours a été éclairé par les interventions de scientifiques passionnés (encadré 3), écologue spécialiste des sols, géologue spécialiste des paléo-environnements et neurobiologiste spécialiste de l'olfaction. Les participants, professeurs d'arts plastiques, de français, de technologie ou de sciences expérimentales ont contribué à la richesse des échanges. Les collègues ont partagé leurs œuvres (encadré 5): peinture, prose, bande dessinée.

L'envie de travailler avec un.e artiste pour un futur projet pédagogique me tente énormément depuis cette formation particulièrement inspirante, afin que mes élèves aient la possibilité de révéler leur potentiel artistique à travers les sciences.

\section{Encadré 2}

\section{S[lab] - Voyage en terres émergées, un dispositif d'enquêtes immersives}

En 2020, l'association S[cube] a décidé de renouveler ses créations afin de favoriser davantage le rapprochement entre sciences et citoyens. Le dispositif conçu, le S[lab], implique le participant physiquement, mentalement et sensiblement dans une expérience singulière. La première thématique abordée dans le S[lab] a été celle, issue du récent rapport du GIEC, de l'usage des terres émergées. Le comité scientifique réuni a identifié trois axes pour aborder les grandes problématiques liées aux sols : la biocénose, les services écosystémiques et les conflits d'usages.

Les constats des médiateurs de l'association quant à l'appropriation des savoirs par les publics visitant les expositions ont conduit à impliquer

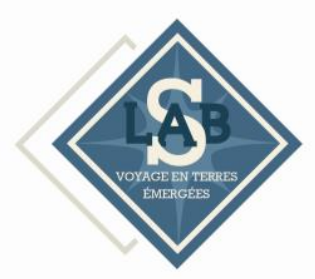
davantage le visiteur dans sa démarche de découverte et d'apprentissage, en proposant un dispositif qui le rende acteur de son cheminement. Le S[lab] est un jeu d'enquêtes immersives qui plonge les visiteurs au cœur d'un mystère qu'ils doivent résoudre en équipe à l'aide d'une tablette. Trois enquêtes traitent de différents enjeux de l'usage des sols : la favorisation de la biodiversité des sols, les conflits d'usages des sols et les services écosystémiques rendus par les sols. Chaque enquête est accessible à des groupes de 1 à 4 personnes dès 5 ans, à condition qu'au moins un des participants soit un lecteur confirmé.

Le raisonnement est libre : les joueurs ont tous les éléments pour résoudre le mystère. Témoins et pièces à conviction leur permettent de construire leur propre parcours pour élucider l'enquête. À la manière de détectives, ils reproduisent des raisonnements scientifiques et adoptent une démarche de concertation. 


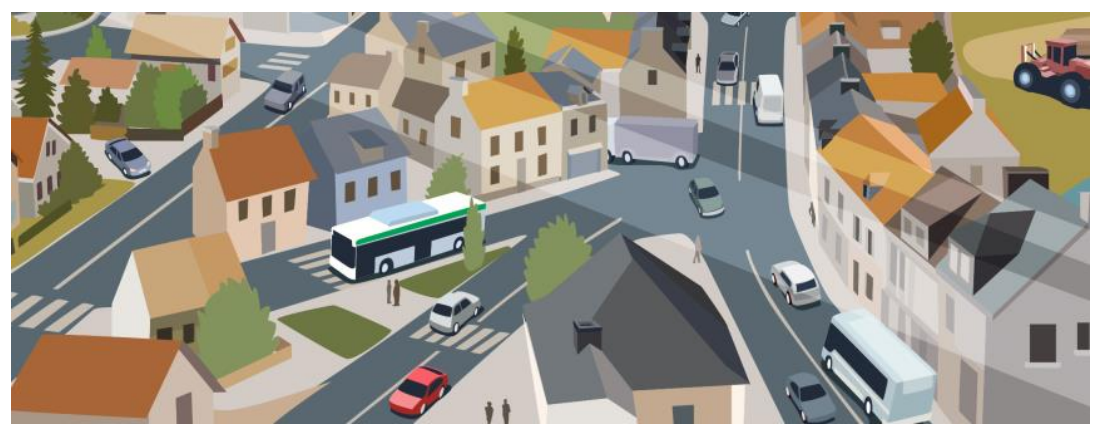

Adapté du jeu "Chronicles of Crime", de David Cicurel, édité par Lucky Duck Games. Projet soutenu par le Ministère de l'Enseignement supérieur, de la Recherche et de I'Innovation et la Région Ile-de-France.

\section{Vous aussi, vous souhaitez jouer?}

Contactez l'équipe S[cube]

Le dispositif se compose d'un décor immersif autoportant permettant de s'adapter à différents lieux.

Selon les enquêtes, le jeu dure de 20 à 60 minutes.

Un avant-goût avec le teaser du jeu : https://youtu.be/6eA3HBpxbYI

\section{Encadré 3}

\section{La parole à Jérémy Jacob, intervenant scientifique}

Géologue et géochimiste, j'effectue mes recherches sur les paléoenvironnements et les paléoclimats. Cela m'amène à dialoguer avec de nombreuses communautés scientifiques : celles des chimistes, des archéologues, des historiens, des urbanistes, des sociologues ou encore des économistes. Par ailleurs, depuis que je suis chercheur, je suis attaché au partage de mon goût pour les sciences. Par exemple, j'ai collaboré avec l'association S[cube] en tant que membre du comité scientifique du projet S[lab] Voyage en terres émergées.

Une approche sensorielle de la science constitue, selon moi, la meilleure voie pour faire résonner des questions scientifiques parfois complexes auprès de tous les publics, qu'il s'agisse des plus jeunes en formation ou de collègues d'autres disciplines. Je l'envisage selon deux aspects : décrire nos objets d'étude par les sens, en complémentarité avec nos outils d'analyse très techniques, et imaginer les paysages sensoriels du passé.

À travers le travail d'Anaïs Tondeur sur l'odeur des sols, la formation Arts et Sciences, un dialogue créatif m'a permis de partager ces idées avec des enseignants du secondaire, de mesurer leur adhésion ou leurs réticences. J'ai également pu me rendre compte que nous partagions des intérêts communs avec Anaïs Tondeur (encadré 4), et qu'il y avait matière à interactions. Nous envisageons une collaboration dans le cadre d'une journée dédiée aux sens en archéométrie, ce champ de l'archéologie qui exploite des techniques empruntées aux sciences expérimentales physico-chimiques ou biochimiques. 


\section{Encadré 4}

\section{La parole à Anaïs Tondeur, intervenante artistique}

Dans une démarche interdisciplinaire, ma pratique artistique prend racine dans la pensée écologique.

Sous la forme d'installations, de photographies, de dessins ou de vidéos, je développe chaque projet dans un processus collaboratif, par le biais de récits spéculatifs ou de protocoles d'investigations. Je travaille ainsi dans une démarche sensible de l'enquête, dans une rencontre avec un territoire et avec d'autres formes de perceptions du monde, comme notamment le long des grandes failles tectoniques avec le géologue Jérôme Fortin, dans une expédition à travers l'océan Atlantique avec l'océanographe Victor Turpin, à travers les sols pollués d'Ile-de France avec l'anthropologue Germain Meulemans, les écologues et pédologues de l'IRD et de l'AgroParistech, sur les sols radioactifs de Tchernobyl avec le philosophe Michael Marder et le bio-généticien Martin Hajduch, dans les profondeurs de Paris avec les géo-techniciens de l'Inspection Générale des Carrières, ou sous les flux atmosphériques de particules fines avec les physiciens de l'atmosphère Rita Van Dingenen et Jean-Philippe Putaud du Centre de recherche de la Commission européenne.

Suite à des études d'art à la Central Saint Martins et au Royal College of Arts à Londres, je me suis progressivement tournée vers les sciences sociales et naturelles, dans le cadre de résidences d'artiste comme à la Commission européenne, au Musée des Arts et Métiers ou au Laboratoire de mécaniques des fluides de l'École Polytechnique.

Mes projets naissent ainsi d'une rencontre avec des contextes multiples de savoirs et compréhensions du monde. Il me semble crucial, dans la période de défis sociétaux et écologiques auxquels nous sommes confrontés, d'associer et de confronter nos différents modes d'être au monde, d'aller puiser dans la multiplicité de nos approches, afin d'inventer puis de donner corps ensemble à d'autres modes d'existence au monde.

Durant la formation, nous avons pu mettre à l'épreuve cette proposition, dans un geste transversal, à partir d'une expérimentation sensible et sensorielle autour de notre relation aux sols (encadrés 1 et 5).

Cet atelier fut également pour moi l'occasion de rencontrer le travail passionnant de l'écologue Stéphane Bazot, du spécialiste de l'olfaction Roland Salesse et du géologue et géochimiste Jérémy Jacob. Je nourris, depuis plusieurs idées de projets autour de la vie et de la respiration des sols, de la pratique du Kodo, une interprétation de la célébration japonaise des champs olfactifs d'un d'arbre ou d'une investigation olfactive de l'histoire géologique de la terre. J'espère un jour développer ces pistes en collaboration avec un ou chacun de ces chercheurs. J'ai commencé à esquisser une piste de collaboration avec Jérémy Jacob (encadré 3), qui serait d'autant plus passionnante que son travail associe deux axes majeurs au cœur de ma pratique : la perception du temps long géologique et l'expérience éphémère d'une odeur. 


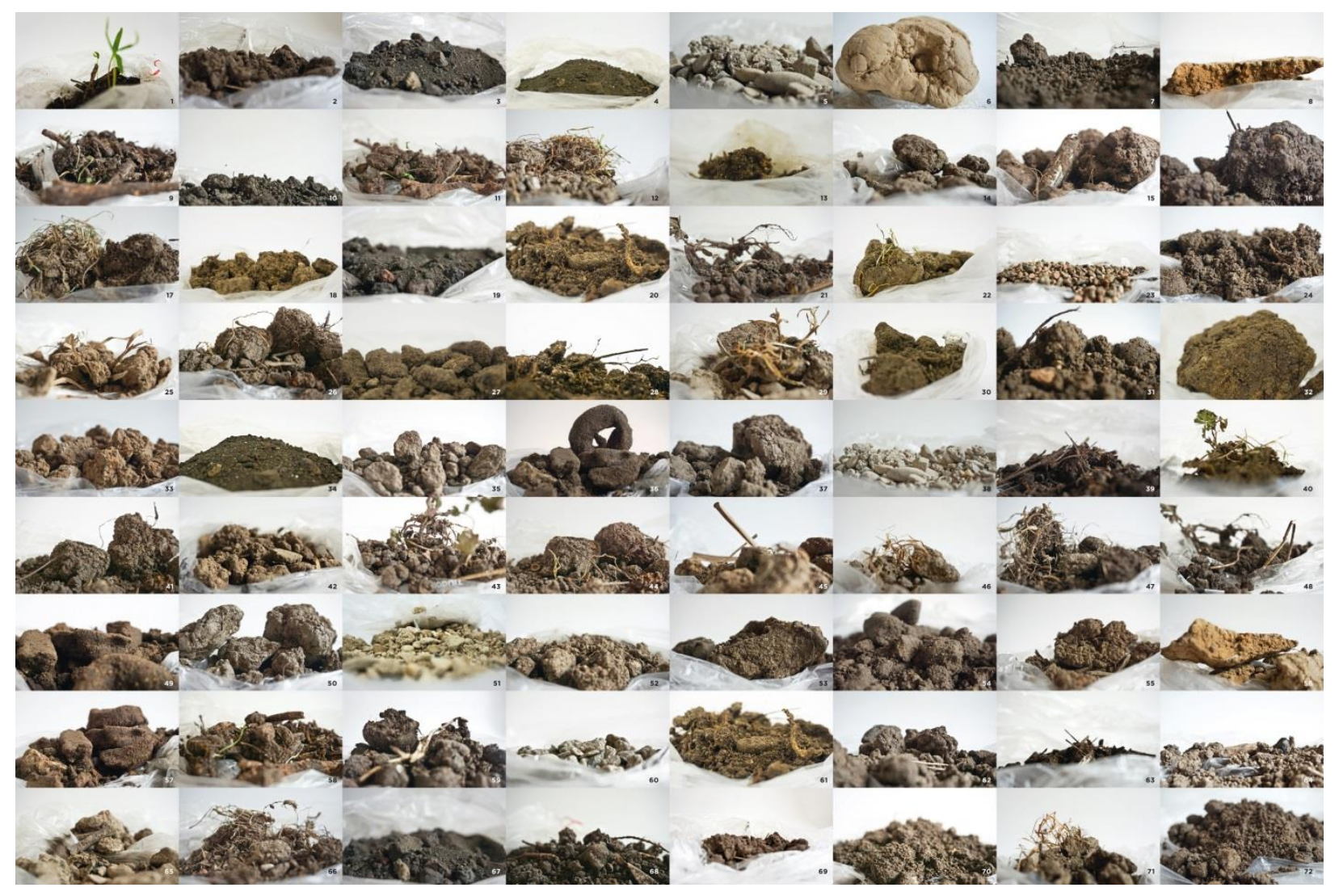

Vue de la collecte des sols, Centre Tinous d'art contemporain, Montreuil, 2018 Pétrichor, Anaïs Tondeur \& Germain Meulemans

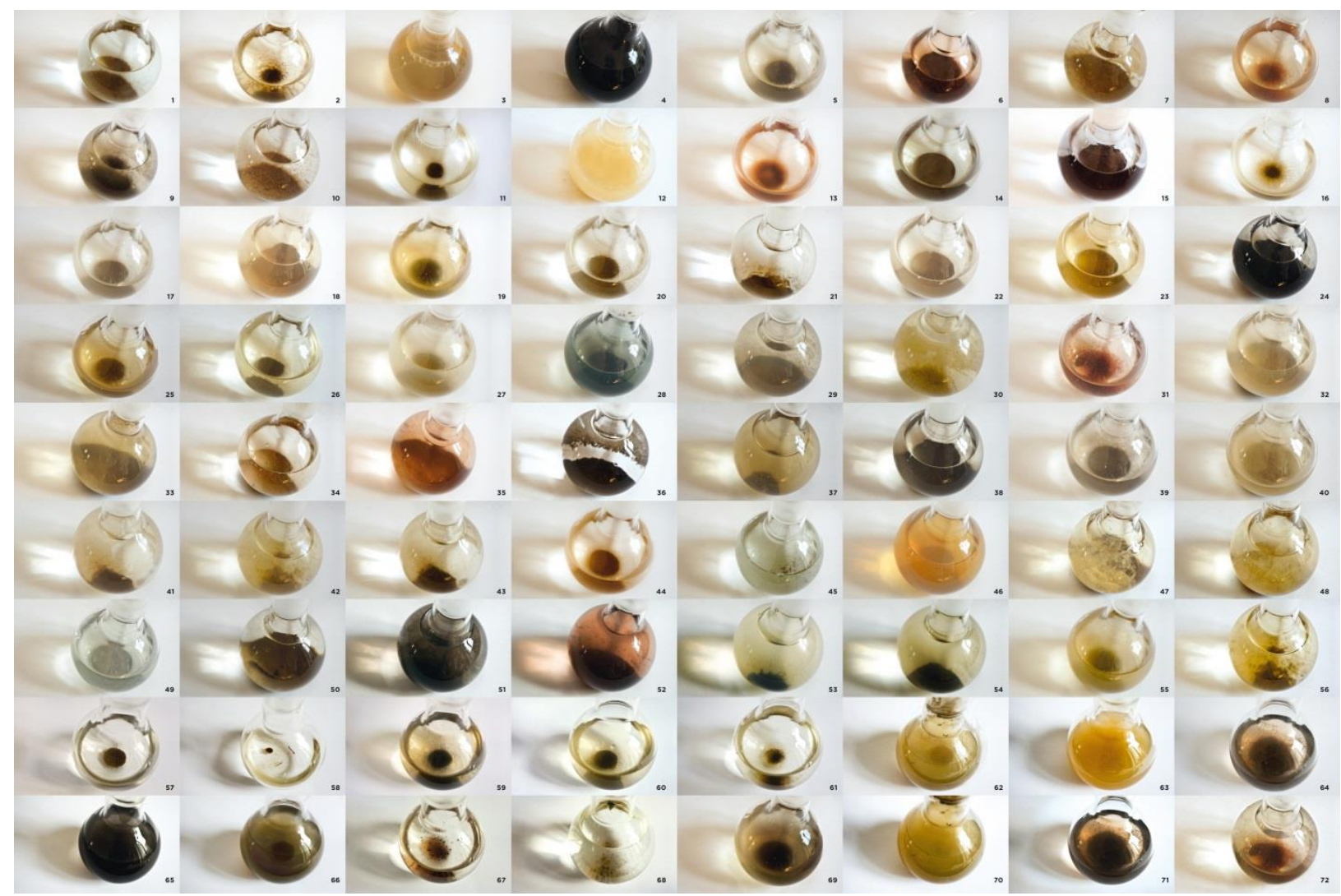

Vue des hydrolats de sols, Centre Tinous d'art contemporain, Montreuil, 2018 Pétrichor, Anaïs Tondeur \& Germain Meulemans 


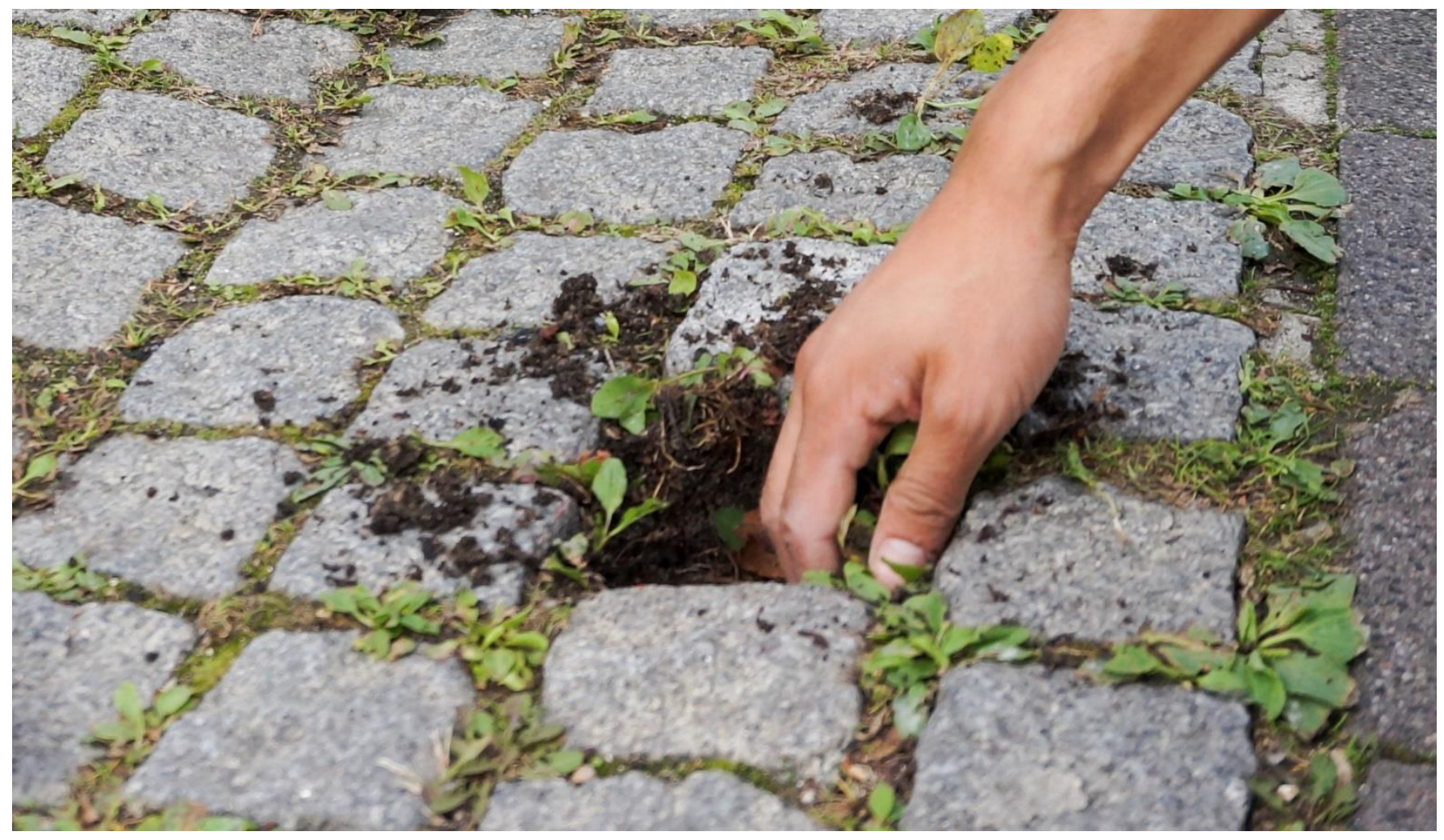

Vue du protocole, Centre Tinous d'art contemporain, Montreuil, 2018

Pétrichor, Anaïs Tondeur \& Germain Meulemans

\section{Encadré 5}

\section{Témoignage de Céline Carrel, professeur en formation}

Professeur d'arts plastiques dans les Hauts-de-Seine, affectée depuis deux ans dans le même collège, je nourris l'idée de construire avec mes collègues d'autres disciplines des projets interdisciplinaires, ce qui a motivé mon inscription à la formation "Arts et sciences, un dialogue créatif".

J'ai été très inspirée par le travail de recherche proposé autour du pétrichor, qui sollicitait l'odorat, un sens rarement utilisé en arts plastiques.

Des prélèvements effectués et des expériences menées, nous avons tiré matière à travailler des questions autour de l'odorat et des autres sens, notamment le toucher, à partir de la matière «terre ». Dans un premier temps, cela m'a évoqué l'idée du souvenir, de la projection dans un autre espacetemps, comme une madeleine de Proust, une émanation d'un moment passé. J'ai donc souhaité travailler sur l'espace dans lequel j'avais réalisé mes prélèvements, aux portes de Paris. En effectuant des recherches, j'ai réalisé que d'anciennes carrières se trouvaient non loin de cet endroit. J'ai voulu imaginer le paysage que j'avais sous les yeux, des années en arrière, à l'époque où les carrières de plâtre à ciel ouvert étaient exploitées. La terre et le plâtre, des matières terrestres, poudrées, volatiles, m'ont amenées à choisir de travailler le pastel sec et le fusain.

Le dessin, par le matériel choisi et l'espace représenté, parle du souvenir de cet espace, revenu en image à travers l'odeur du pétrichor. 


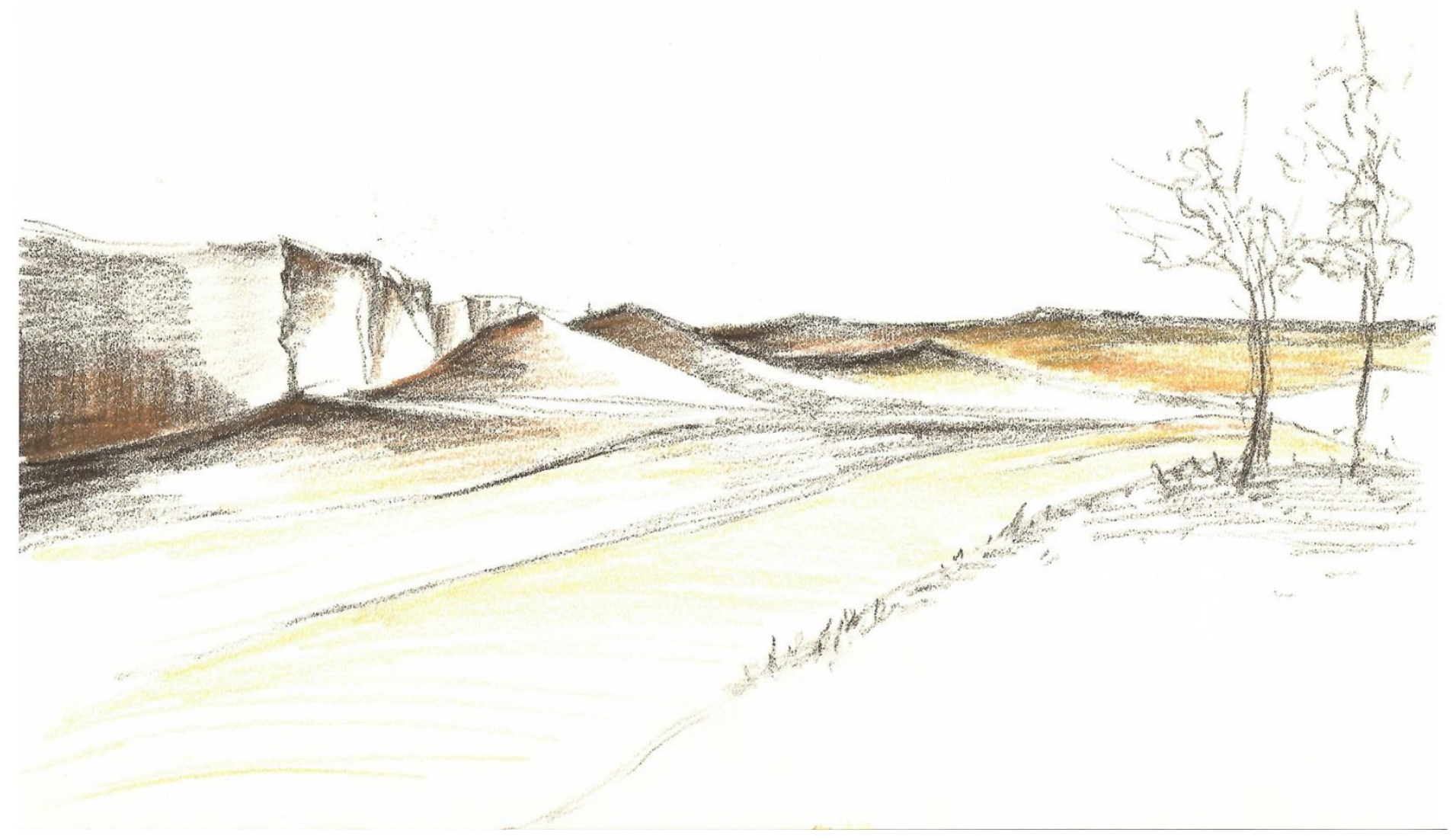

Illustration : Céline Carrel, 2021

\section{Liens bibliographiques Anaïs Tondeur}

Soil Fictions: Addressing Urban Soils between Art, Soil Ecology, and Anthropology. Collaborative An- thropologies, Germain Meulemans, Marine Legrand, Anaïs Tondeur, Yesenia Thibault-Picazo, Alan Vergnes. University of Nebraska Press, 2018, 10 (1-2), pp.20-44. 10.1353/cla.2017.0001 . hal- 02882883

Paris flotte-t-il ? Musée des Arts et Métiers, Germain Meulemans, Anaïs Tondeur https://www.arts-et-metiers.net/sites/arts-et-metiers.net/files/asset/document/paris_flotte-t-il_online.pdf

Pétrichor ou l'odeur des terres de Montreuil, Centre Tignous d'Art Contemporain, Germain Meulemans, Anaïs Tondeur https://fr.scribd.com/document/446023665/Petrichor-Edition-1-2 https://fr.scribd.com/document/446023591/Petrichor-Edition-2-2

FAIRNESS, Resonnance II, Joint Research Centre

This book is a snapshot of the road the Joint Research Centre set out to follow bringing together art, science and society. It shows the experimental character and uncertainties of our endeavour, sometimes the divergences of opinions or the difficulties of working with different frames of mind - or should we say different bodies of knowledge?

https://fr.scribd.com/document/453802895/kjna28727enn

\section{Liens bibliographiques intervenants scientifiques}

Violaine de Carné https://www.tiretlalyre.com/compagnie-violainedecarne

Roland Salesse https://www.quae.com/auteur/1607/roland-salesse 


\section{Liens bibliographiques Arts et Sciences}

Art + science / Wilson, Stephen ; Berton, Gilles. Trad. - Paris: Ed. Thames \& Hudson, 2010 - 208 p. - Bibliogr. ; Webogr.; Index

Art et science / Strosberg, Eliane. - Paris : Editions UNESCO, 1999 - 245p. ; in- 4 ; Ill.

Entre arts et sciences / Bordeaux, Marie-Christine. Dir. ; Fourmentraux, Jean- Paul ; Martin-Juchat, Fabienne ; Gentès, Annie ; Jutant, Camille ; Sevin, Jean- Christophe ; Vidal, Geneviève ; Cordonnier, Sarah ; Villagordo, Eric. - In : Culture et musées, 2012, n 19 - Avignon : Actes Sud, 2012 - 207 p. ; Ill. - Résumés des articles en français, anglais et espagnol; - Bibliogr.; - Présentation des auteurs; - Revue : numéro spécial

Laboratoires de l'art = Art laboratories : [Exposition. Musée des arts et métiers, 10 mai - 4 septembre 2016]

La Science n'EsT pas l'art : brèves rencontres / Lévy-Leblond, Jean-Marc. - Paris : Hermann, 2010 - 119 p. ; In-6 ; Ill. en noir et en coul.

Maths \& arts plastiques / Cohen, Gilles, Dir. - Paris : Pole, 2005. - Tangente H.S., n²3. - 157 p. : Ill. ; In-8. - Bibliogr. Revue numéro spécial

ONS. Un Modèle pour des collaborations possibles entre art et science / Paysant, Michel. Dir. ; Le Neün, Corinne. Dir. Paris : Ecole Nationale Supérieure d'Arts de Paris Cergy, 2014 - 319 p. ; In-8 ; - ONS = Objets non standard, programme de recherche mené de 2011 à 2013, conclu par une exposition à l'YGREC, espace d'exposition de l'ENSAPC (Paris 13e), janvier-février 2013

Quand l'art rencontre la science / Ameisen, Jean Claude ; Brohard, Yvan. - Paris : Editions de la Martinière, 2007 - 143 p. ; In-8; Ill en coul. 